\title{
Absorber's Effect Projected Directly Above Improves Spatial Resolution in Near Infrared Backscattered Imaging
}

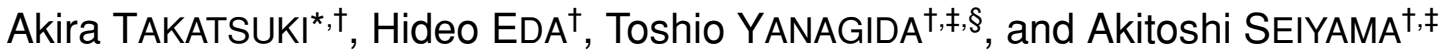 \\ * Department of Biophysical Engineering, Graduate School of Engineering Science, \\ Osaka University, Toyonaka, 560-8531 Japan; \\ † Brain Information Group, Kansai Advanced Research Center, Communications Research Laboratory, \\ Kobe, 651-2492 Japan; \\ ₹Department of Physiology and Biosignaling, Graduate School of Medicine, \\ Osaka University, Suita, 565-0871 Japan; and \\ $\S$ Laboratories for Nanobiology, Graduate School of Frontier Biosciences, \\ Osaka University, Suita, 565-0871 Japan
}

\begin{abstract}
The spatial resolution of near infrared spectroscopic imaging in brain function mapping studies needs to be improved. Most near infrared spectroscopic imaging systems use optical fibers that are arranged like a lattice. The light source and the detector have one-on-one correspondence at intervals of about $3 \mathrm{~cm}$. In this study, we apply several detectors against one source to improve spatial resolution. We assume that a local absorber within a strong scattering medium is a model for local cerebral activation. When we calculate absorbance, which is used to spectroscopically calculate hemoglobin concentration, the peak position shifts away from above the position where an absorber is located. As far
\end{abstract}

as absorbance is calculated, we cannot obtain spatial information about the absorber even if many detectors are used against one source. When we calculate the difference between detected light intensities, however, we demonstrate that an absorber projected onto the measuring surface has an influence directly above it. We predict this property from a light diffusion equation and also prove it experimentally through measurements of a uniform resinous phantom with an absorber. This is one of the basic principles supporting the achievement of higher spatial resolutions with near infrared spectroscopic imaging. [The Japanese Journal of Physiology 54: 79-86, 2004]

Key words: near infrared light, backscattered imaging, spatial resolution, polyacetar phantom, brain science.

Near infrared spectroscopic imaging has become one
of the most powerful tools in the study of human brain
functions. It can detect human brain activations nonin-
vasively because near infrared light transmits living
tissue well [1]. Transmitted and scattered light can be
detected at points several centimeters away from the
injection point after near infrared light has been di-
rected onto the scalp. Part of the detected light passes
through the brain cortex [2]. We can calculate changes
in oxygenated hemoglobin concentrations and deoxy-
genated hemoglobin concentrations that are caused by
brain activity because hemoglobin has characteristic absorption spectra. Human brain functions measured by near infrared light obtained by use of a pair of light source and detector probes were reported in 1993 by Chance et al., Villringer et al., Hoshi and Tamura, and Kato et al. [3-6]. Subsequently, researchers using multiple probes reported diffuse tomographic imaging [7-10].

Present human brain function studies use a near infrared spectroscopic imaging system that visualizes hemodynamic changes in the cortex as a topographic image. This system makes topographic images from data sets measured through spatially set multiple

Received on December 16, 2003; accepted on February 6, 2004

Correspondence should be addressed to: Hideo Eda, Brain Information Group, Kansai Advanced Research Center, Communications Research Laboratory, 588-2 Iwaoka, Iwaoka-cho, Nishi-ku, Kobe, 651-2492 Japan. Tel: +81-78-969-2276, Fax: +81-78-969-2279, E-mail: eda@po.crl.go.jp 


\section{A. TAKATSUKI et al.}

probe pairs [11-13]. Many basic studies on brain functions have been done with the near infrared spectroscopic imaging system. For example, this system detected detailed hemodynamic changes in the primary cortex $[14,15]$ and could be used for simultaneous recording with magnetic resonance imaging (MRI) [16-18]. Moreover, research that could only have been carried out with a near infrared spectroscopic imaging system has been reported. For example, the system has been used to detect brain activation during gait [19], to measure the brain activation of infants [20-22], and to simultaneously record with transcranial magnetic stimulation (TMS) [23]. When the system is used, the light source and the detector are placed apart at intervals of about $3 \mathrm{~cm}$. It is believed that the ratio of light passing through the brain cortex is low when the interval is less than $3 \mathrm{~cm} \mathrm{[24,}$ 25]. The spatial resolution of near infrared spectroscopic imaging has been fixed by this probe interval distance.

In brain function mapping studies, however, improved spatial resolution is needed. Studies that have aimed at improving the spatial resolution of near infrared spectroscopic imaging have been reported. Yamamoto et al. multiplied the density of probe pairs and improved the accuracy of spatial information [26]. However, the practice of one data item being represented by one probe pair at an interval of about $3 \mathrm{~cm}$ has remained unchanged.

In this study, our aim was to detect the absorber's position independent of probe interval distances. We introduced an approach with multiple detectors against one source and not the conventional probe pair, which consisted of a single detector against one source. We assumed that changes in local absorption occurred within a strong scattering medium in a model of human brain activation at the cerebral cortex. We discuss this theoretically from the viewpoint of a light diffusion equation and also experimentally from the measurement of a resinous phantom with an absorber.

Light diffusion equation. During optical measurement, light is injected from a point source to a medium, and changes in light signal at a detector are measured. Light propagation in tissue with strong scattering can be described through a light diffusion equation [27]. The contribution of internal optical properties to the signal, in other words, the photonmeasurement density function (PMDF), has been investigated $[25,28,29]$. This corresponded to the change in signal that is caused by moving the absorber's position in the medium when the source and the detector are fixed. In our study, we fixed the posi- tions of the source and absorber first and considered how the absorber influenced signals at the measuring surface. This corresponded to the contribution of signals detected by multiple detectors against one source. This is another model that is different from PMDF.

Farrell et al. derived an analytical solution of light diffusion equation to the semi-infinite condition [27]. The geometry of the model is illustrated in Fig. 1, and the origin of the coordinates is defined as the source position on the medium. The $\rho$ axis is taken as a line parallel to the measuring surface, and the $z$ axis is taken as a line directed directly below the source. We applied an extrapolated boundary condition, which satisfied the condition that the summation of a positive real source and a negative image source was zero. Fluence rate $\phi$ at point $(\rho, z)$ in the medium is described by Eqs. 1-8 [27].

$$
\phi(\rho, z)=\frac{1}{4 \pi D} \cdot\left(\frac{\exp \left(-\mu_{\mathrm{eff}} \cdot r_{\mathrm{p}}\right)}{r_{\mathrm{p}}}-\frac{\exp \left(-\mu_{\mathrm{eff}} \cdot r_{\mathrm{n}}\right)}{r_{\mathrm{n}}}\right)
$$

where $r_{\mathrm{p}}$ is the distance between the positive source and point $(\rho, z)$, and $r_{\mathrm{n}}$ is the distance between the negative source and point $(\rho, z)$.

$$
r_{\mathrm{p}}=\sqrt{\left(z-z_{0}\right)^{2}+\rho^{2}}
$$

and

$$
r_{\mathrm{n}}=\sqrt{\left(z+z_{0}+2 z_{\mathrm{b}}\right)^{2}+\rho^{2}}
$$

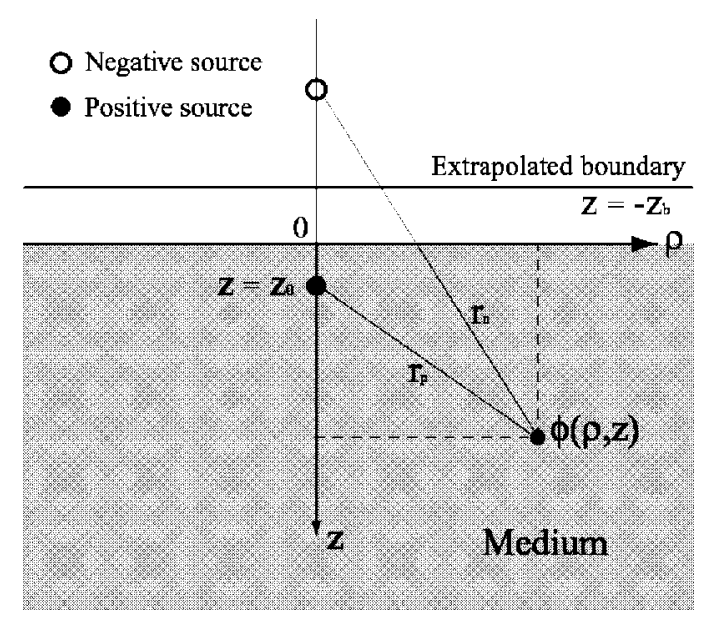

Fig. 1. Concept of light propagation in strong scattering medium in semi-infinite model. Fluence rate in the medium is calculated by extrapolated boundary. The extrapolated boundary is a zero boundary, which is the sum of a positive and negative source. The position of an extrapolated boundary that has a zero value is $z_{b}$. The distance between a positive source and a point $(\rho, z)$ is $r_{\mathrm{p}}$, and the distance between a negative source and a point $(\rho, z)$ is $r_{\mathrm{n}}$. 


$$
\begin{gathered}
\mu_{\mathrm{eff}}=\sqrt{3 \mu_{\mathrm{a}} \cdot \mu_{\mathrm{s}}{ }^{\prime}} \\
z_{0}=\frac{1}{\mu_{\mathrm{s}}{ }^{\prime}} \\
z_{b}=2 A D \\
A=\left(1+r_{d}\right) /\left(1-r_{d}\right)
\end{gathered}
$$

and

$$
r_{\mathrm{d}}=-1.440 n_{\mathrm{rel}}^{-2}+0.710 n_{\mathrm{rel}}^{-1}+0.668+0.0636 n_{\mathrm{rel}}
$$

where $\mu_{\text {eff }}$ is the effective attenuation coefficient, $\mu_{\mathrm{a}}$ is the absorption coefficient, $\mu_{\mathrm{s}}{ }^{\prime}$ is the reduced scattering coefficient, $z_{\mathrm{b}}$ is the distance of extrapolated boundary from the origin, and $n_{\text {rel }}$ is the relative refractive index of the medium-air interface.

$$
D=\frac{1}{3 \mu_{\mathrm{s}}{ }^{\prime}}
$$

where $D$ is the diffusion constant, which contains only $\mu_{\mathrm{s}}{ }^{\prime}$ [30]. In reflectance measurement, the signal intensity, which is proportional to the fluence rate at the surface, can be measured experimentally.

We considered an inhomogeneous case, which has an absorber in high scattering media, as the model for local cerebral activations. Figure 2 has an illustration of the model. In this paper, we made two assumptions. The first was decreased fluence rate because of the absorber at position $\left(\rho_{\text {absorber }}, z_{\text {absorber }}\right)$, which was defined as "Dark fluence rate," and described by Eq. 10.

$$
\text { Dark fluence rate }=\text { dark_fr }\left.(\rho, 0)\right|_{\operatorname{Absorber}\left(\rho_{\text {absorber }}, z_{\text {absorber }}\right)}
$$

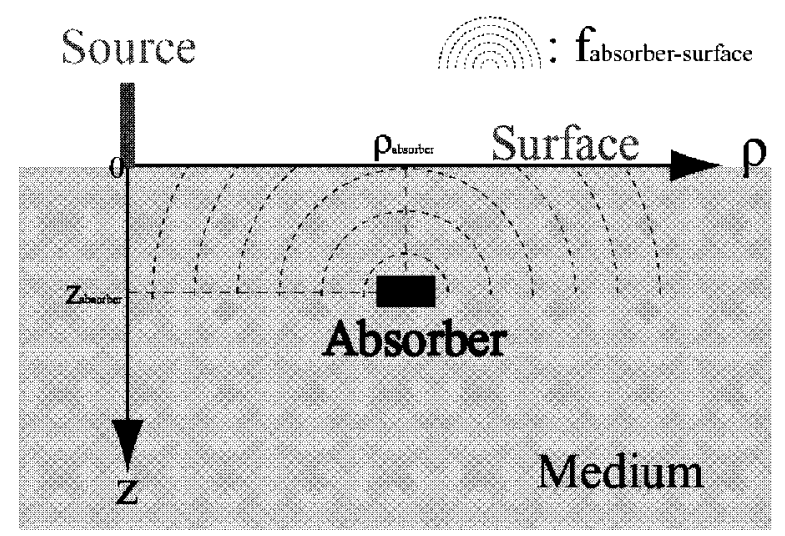

Fig. 2. An absorber ( $\left.\rho_{\text {absorber }}, z_{\text {absorber }}\right)$ produces a dark fluence rate. The origin of the $\rho-z$ plane is set as the source position. Dark fluence rate is calculated as a multiplication fluence rate at the absorber position and the transfer function from an absorber to the measuring surface. The circular dotted curves indicate $f_{\text {absorber-surface, }}$ which is the transfer function from an absorber to the measuring surface.
The second was measurable fluence rate $\Phi(\rho)$ at the measuring surface under the case with the absorber equaled the sum of the fluence rate $\phi(\rho, 0)$ under the case without an absorber and "Dark fluence rate," dark_fr $(\rho, 0)$. This assumption was derived from firstorder born approximation to the light diffusion equation. Our assumptions are described in Eq. 11.

$$
\Phi(\rho)=\phi(\rho, 0)+\text { dark_fr }\left.(\rho, 0)\right|_{\text {Absorber }\left(\rho_{\text {absorber }}, z_{\text {absorber }}\right)}
$$

The light diffusion equation was basically applied only to the homogeneous medium. In this study, however, we assumed that the light diffusion equation would be able to be applied to calculate the influence of local absorbance change within the medium had on signals at the measuring surface.

"Dark fluence rate" in the medium can be calculated as follows. First, we should calculate the fluence rate that reaches the absorber and how much is absorbed by it. Next, let us consider by using the light diffusion equation how the absorber's influence propagates to the measuring surface. We define the fluence rate at the absorber's position as $\phi\left(\rho_{\text {absorber }}, z_{\text {absorber }}\right)$ and the transfer function from the absorber to the measuring surface as $f_{\text {absorber-surface. "Dark fluence }}$ rate," at the detector position is given by Eq. 12 .

$$
\begin{aligned}
& \text { dark_fr}\left.(\rho, 0)\right|_{\text {Absorber }\left(\rho_{\text {absorber }}, z_{\text {absorber }}\right)} \\
& \quad=-\phi\left(\rho_{\text {absorber }}, z_{\text {absorber }}\right) \cdot f_{\text {absorber-surface }} \cdot k
\end{aligned}
$$

where $k$ is a constant, including the absorbance coefficient of the absorber and optical path length in the absorber. Equation 13 is derived according to Eqs. 11 and 12 .

$$
\begin{aligned}
\Phi(\rho) & =\phi(\rho, 0)+\left.\operatorname{dark} \operatorname{fr}(\rho, 0)\right|_{\text {Absorber }\left(\rho_{\text {absorber }}, z_{\text {absorber }}\right)} \\
& =\phi(\rho, 0)-\phi\left(\rho_{\text {absorber }}, z_{\text {absorber }}\right) \cdot f_{\text {absorber-surface }} \cdot k
\end{aligned}
$$

We calculated Abs, which is a logarithmic calculation, and Diff, which is subtraction as the absorber's effect. Abs was calculated by the use of Eq. 14 .

$$
\begin{aligned}
& \text { Abs }=-\log _{10}\left(\frac{\Phi(\rho)}{\phi(\rho, 0)}\right) \\
& =-\log _{10}\left(\frac{-\phi\left(\rho_{\text {absorber }}, z_{\text {absorber }}\right) \cdot f_{\text {absorber-surface }} \cdot k}{\phi(\rho, 0)}+1\right)
\end{aligned}
$$

Diff was calculated by the use of Eq. 15. 


\section{A. TAKATSUKI et al.}

$$
\begin{aligned}
\operatorname{Diff} & =\Phi(\rho)-\phi(\rho, 0) \\
& =-\phi\left(\rho_{\text {absorber }}, z_{\text {absorber }}\right) \cdot f_{\text {absorber-surface }} \cdot k
\end{aligned}
$$

$\phi(\rho, 0)$ has spatially spreading values, which are exponentially decaying from the light source position. The solution to Eq. 14 includes $\phi(\rho, 0)$, but that of Eq. 15 does not include $\phi(\rho, 0)$. This does not affect the spatial information of either Abs or Diff when the positions of the source and the detector are fixed for PMDF. However, this has a bad influence on the spatial information of Abs when the positions of the detector are not fixed. We expected that spatial information on the absorber would be concealed in the Abs calculation because of $\phi(\rho, 0)$ [31]. On the contrary, Diff is calculated, the absorber's effect propagates to the measuring surface directly above it in manner of diffusion. Because diffusion is a function of distance, attenuation is small when the distance is short. The position on the measuring surface, which has the shortest distance between the absorber and measuring surface, is directly above the absorber. Consequently, we expected that the absorber would affect projection to the measuring surface directly above the absorber when the difference between the detected light intensities was calculated.

\section{METHODS}

We used the near infrared optical imaging system (OPTIM_A) developed by our group [13, 16]. This system has 12 optical source probes bundling three laser diodes $(780,805$, and $830 \mathrm{~nm})$ and 12 detector probes leading to the photo multiplier tubes. The diameter of both probes is $2 \mathrm{~mm}$. We generally placed these probes in latticed positions to acquire topographic images. The system could acquire topographic functional images of relative changes in oxygenated, deoxygenated, and total hemoglobin concentrations. We could use it not only in clinical use, but also in basic optical-measurement studies by using a subset of its function. In this study, we used only one probe pair from this system, and we used a $780 \mathrm{~nm}$ wavelength light-source and a constant gained photomultiplier.

We prepared a phantom as the measuring object (Fig. 3). It was made of white resin (polyacetar, absorption coefficient: $0.001 \mathrm{~mm}^{-1}$; reduced scattering coefficient: $1 \mathrm{~mm}^{-1}$ ) that had a scattering characteristic comparable to living tissue. It consisted of a fixed body and a slidable slab. The slider was $10 \mathrm{~mm}$ thick and slid horizontally at a depth $10 \mathrm{~mm}$ from the measuring surface. It had a tablet pocket, which was a hole $10 \mathrm{~mm}$ in diameter. We made white and black

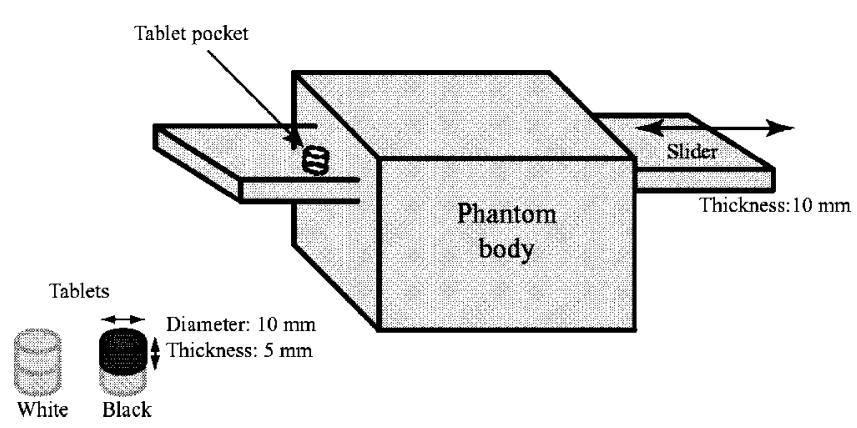

Fig. 3. Diagram of phantom for measuring object. The phantom consists of a body and a slider. The slider is $10 \mathrm{~mm}$ thick. It has a tablet pocket $10 \mathrm{~mm}$ in diameter. The body, slider, and white tablet have the same optical properties. The black tablet is an absorber. The tablets are $10 \mathrm{~mm}$ in diameter and $5 \mathrm{~mm}$ thick.

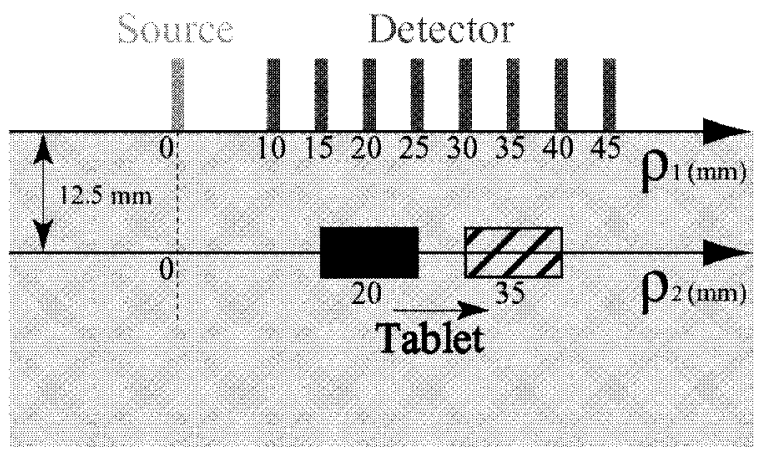

Fig. 4. Experimental scheme to measure phantom. The detector positions are 10, 15, 20, 25, 30, 35, 40, and $45 \mathrm{~mm}$ on the $\rho_{1}$ axis, and the tablet positions are 20, 25, 30 , and $35 \mathrm{~mm}$ on the $\rho_{2}$ axis, which is $12.5 \mathrm{~mm}$ beneath the $\rho_{1}$ axis.

tablets, which were cylinders $10 \mathrm{~mm}$ in diameter and $5 \mathrm{~mm}$ in thickness. The white tablet was made of the same resin as the body, and the black tablet was the absorber. Thus we could place the tablet at the position being aimed at.

Figure 4 shows the experimental scheme. The $\rho_{1}$ axis is taken as the line where the detector is placed, and the origin of this axis is defined as the source position. The $\rho_{2}$ axis is also taken as a line parallel to the $\rho_{1}$ axis that passes through the center of the tablet, and the origin of the $\rho_{2}$ axis is defined as being directly below the source. The experiment was done as follows. First we placed the white tablet into the tablet pocket in the slider. We placed the detector on the phantom $10 \mathrm{~mm}$ away from the source and detected light intensity. The detector was placed at $5 \mathrm{~mm}$ steps away from the source, and the light intensity was measured at 8 points $(10,15,20,25,30,35,40$, and $45 \mathrm{~mm}$ on $\rho_{1}$ axis). The detected light intensities were defined as white tablet data for reference. Next we placed the black tablet into the tablet pocket. The cen- 
ter of the black tablet was placed at a depth of $12.5 \mathrm{~mm}$ from the measuring surface. We repeated the sequence of measurements under four black tablet conditions $\left(20,25,30\right.$, and $35 \mathrm{~mm}$ on $\rho_{2}$ axis). We then obtained the data sets under all conditions as black tablet data.

\section{RESULTS}

We plotted the detected light intensity at each detector position when the tablet was positioned $25 \mathrm{~mm}$ on the $\rho_{2}$ axis. Figure 5(a) shows this in logarithmic scale, and Fig. 5(b) shows it in linear scale. The plot indicates the reference data when the white tablet was used. As the source-detector distance is wider, detected light intensity decreased exponentially. The detected light intensity with the black tablet is smaller than that of the white tablet.

We detected light intensity at each detector position and at each tablet position. We calculated "Abs" and "Diff" as the absorber's effect from the data sets for the white and black tablets.

We calculated the Abs with Eq. 16.
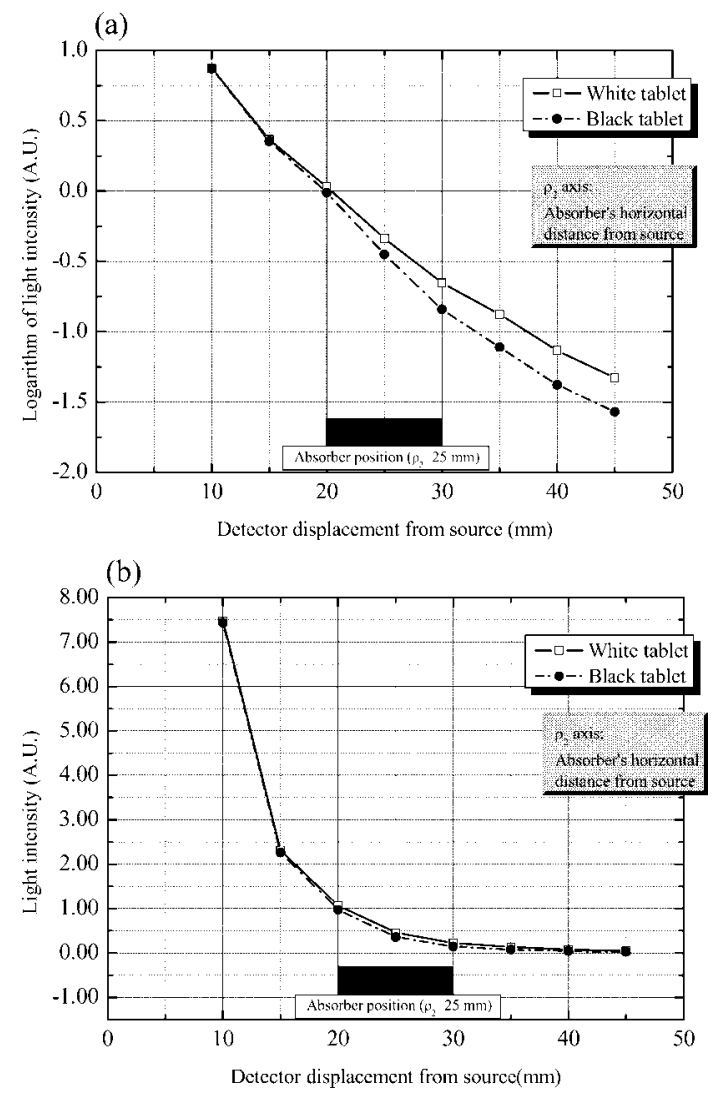

Fig. 5. Light intensity with white or black tablets. The black tablet is $25 \mathrm{~mm}$ on $\rho_{2}$ axis as an absorber. (a) Light intensity is indicated by a logarithmic scale. (b) Light intensity is indicated by a linear scale.

$$
\text { Abs }=-\log _{10}\left(\frac{\text { black tablet data }}{\text { white tablet data }}\right)
$$

This calculation corresponds to the differences between the two curves in Fig. 5(a). Figure 6(a) shows that the peak for the absorber's effect in Abs shifts away from above the black tablet. We also calculated Diff with Eq. 17.

$$
\text { Diff }=\text { black tablet data }- \text { white tablet data }
$$

This calculation corresponds to the differences between the two curves in Fig. 5(b). Figure 6(b) shows that the peak for the absorber's effect in Diff appears directly above the black tablet.

Figure 7 plots Diff data sets under each tablet position $\left(20,25,30,35 \mathrm{~mm}\right.$ on $\rho_{2}$ axis). The peak intensity decreases as the distance between the source and tablet increases.

\section{DISCUSSION}

When we produce images from spatially multiple measured data sets to establish where the absorber is, the peak for the data should indicate the absorber's position. Figure 6(a) shows that the peak position for
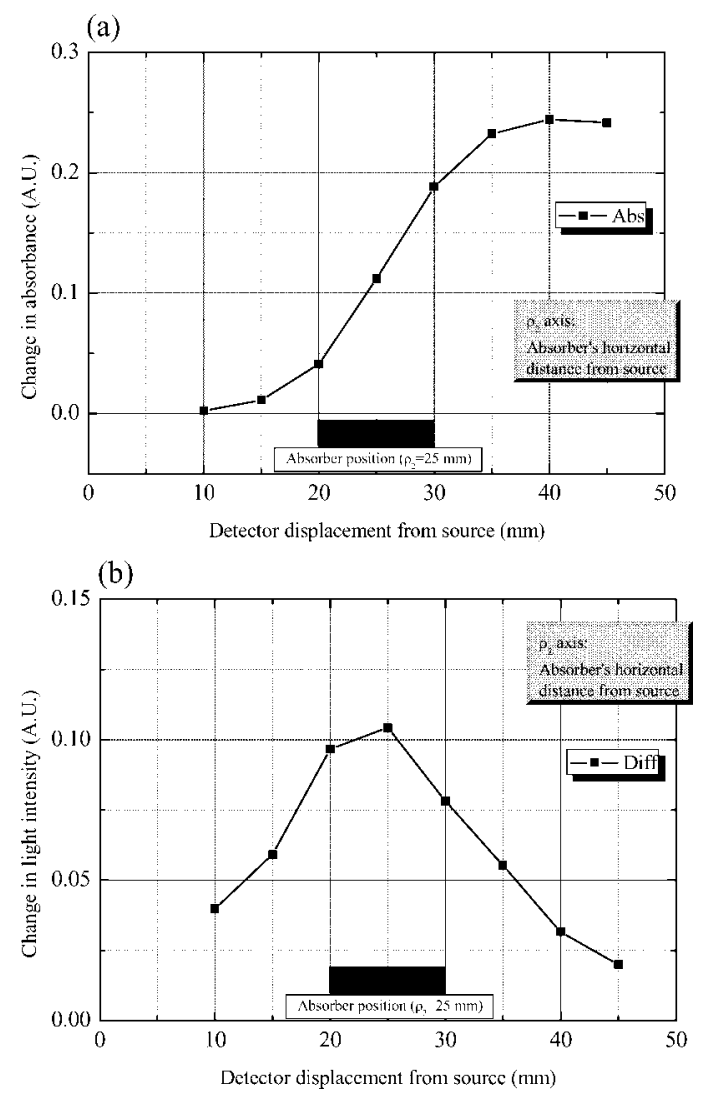

Fig. 6. Differences with and without absorber. (a) Abs indicates change in absorbance. (b) Diff indicates change in light intensity. 
Abs shifts away from above the absorber because of the spatial distribution in the reference data. This means that as far as Abs is calculated, we cannot know where the absorber is. It is widely known that light pathways between the light source and the detector within a medium have a banana shape. When the positions of the source and detector are fixed, the midpoint between them has the best sensitivity with or without an absorber. This is a different model from this study's in that the optimal detection point is sought after the position of the source and the absorber is fixed. When we fix source and absorber positions and calculate Abs, we can only say that the optimal detector position shifts from above the absorber. In reflectance imaging, estimating the absorber's position by calculating Abs is very difficult.

We demonstrated that the Diff absorber's effect appears directly above the absorber from theoretical and experimental results in Fig. 6(b). This means that the peak for measured data indicates that the absorber's position is in contrast to Abs. In an estimation of the absorber's position, Diff has an advantage over Abs, which is conventionally calculated for hemoglobin concentration changes. Improved spatial resolution is expected when we use multiple detectors against one source and calculate Diff.

As we can see in Fig. 7, the absorber's effect appears directly above the absorber in calculating Diff regardless of source-detector distances. We divided the curve for Diff in Fig. 7 by the value measured when the detector is placed above the absorber. After this standardization, we overlapped the four curves in Fig. 8 as the relative positions between the absorber and the detector positions were the same. The shapes of the four curves in Fig. 8 acquired from different source-detector distances are approximately consistent. Diff is calculated as $-\phi\left(r_{\text {absorber }}, z_{\text {absorber }}\right) \cdot f_{\text {absorber- }}$ source $k$ in the light diffusion equation. We can interpret that this equation has a light source whose fluence rate is $-\phi\left(r_{\text {absorber }}, z_{\text {absorber }}\right) \cdot k$ and the diffusion from the source is represented as $f_{\text {absorber-source. }}$ According to this interpretation, $-\phi\left(r_{\text {absorber }}, z_{\text {absorber }}\right) \cdot k$ is proportional to the peak for the curve in Fig. 7, and $f_{\text {absorber-source }}$ corresponds to how much the curve in Fig. 8 is blurred. Constant curve blur independent of the source-detector distance is expected from the light diffusion equation when the depth of absorber $z_{\text {absorber }}$ is constant. Figure 8 indicates that the experimental data are consistent with this theoretical expectation.

The curves in Fig. 8 are more expanded than the actual size of the absorber. The image of the absorber has blurred because light is scattered. From the experimental data in Fig. 8, we defined the full width at

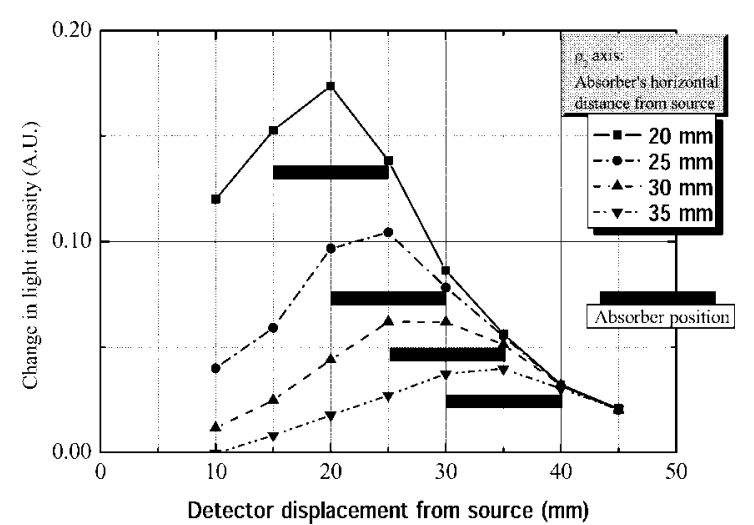

Fig. 7. Difference in light intensity under four absorber positions. An absorber is at $20,25,30$, or $35 \mathrm{~mm}$ on the $\rho_{2}$ axis.

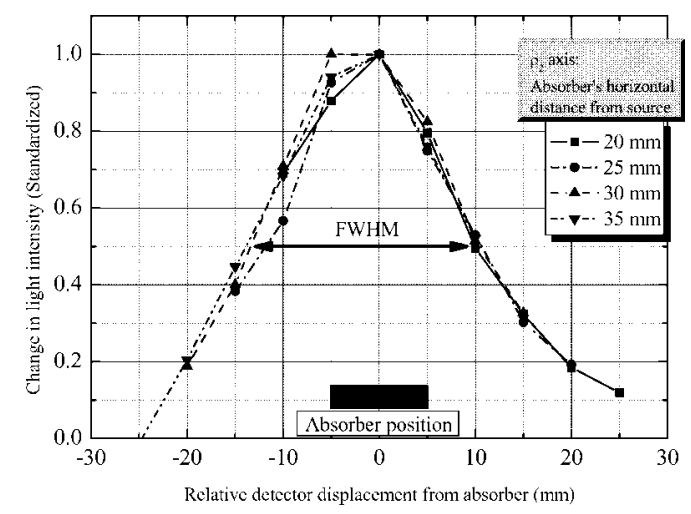

Fig. 8. Standardization of difference in light intensity under four absorber positions. Four curves for relative distance between absorber and detector positions are plotted as absorber positions are centered. The FWHM of four curves are approximately the same.

half-maximum (FWHM) of the curve as the degree of blurring. Under these experimental conditions, we observed an absorber with an actual diameter of $10 \mathrm{~mm}$ as being about $23 \mathrm{~mm}$ at FWHM. Here we defined the spatial resolution as $13 \mathrm{~mm}$, which was derived by subtracting $10 \mathrm{~mm}$ from $23 \mathrm{~mm}$. We demonstrated that we could effectively obtain spatial information different from the conventional measurement method that had only one data item per about $30 \mathrm{~mm}$. Functional MRI (fMRI) has the highest spatial resolution of all current noninvasive brain function measuring equipment. MRI certainly acquires functional images in the $\mathrm{mm}$-order scale. However, in many cases, the images need to be smoothed in preprocessing analysis. The effective spatial resolution of fMRI data depends on the Gaussian kernel size used for smoothing. Worsley and Friston discussed it and used kernel sizes of 4, 8, and $16 \mathrm{~mm}$; they recommended that smoothness should be at least twice the voxel size [32]. It can be said that an effective spatial resolution of fMRI data 
for analysis is effectively about $1 \mathrm{~cm}$. Compared to fMRI, our results indicate that near infrared spectroscopic measurements satisfied the values required by noninvasive human brain function studies.

Present human brain function studies, in other words human brain function mapping studies, have tended to attach importance to obtaining the position of activation instead of determining the quantity of activation. We need studies like this one that are oriented toward obtaining detailed positions for activation to obtain clearer findings on human brain function studies. When we use multiple detectors against one source and calculate Diff, we can directly obtain the position of the absorber. This property is one of the basic principles in improving the spatial resolution of near infrared spectroscopic imaging in studies on human brain functions.

We would like to thank Dr. I. Sase, Dr. T. Murata, and the other brain information group members for their critical discussions. This work was supported in part by a Research Fellowship Grant (JSPS-H13-DC1-01120) from the Japan Society for the Promotion of Science for Young Scientists.

\section{REFERENCES}

1. Jobsis FF: Noninvasive, infrared monitoring of cerebral and myocardial oxygen sufficiency and circulatory parameters. Science 198: 1264-1267, 1977

2. Delpy DT, Cope M, van der Zee P, Arridge S, Wray S, and Wyatt J: Estimation of optical pathlength through tissue from direct time of flight measurement. Phys Med Biol 33: 1433-1442, 1988

3. Chance B, Zhuang Z, UnAh C, Alter C, and Lipton L: Cognition-activated low-frequency modulation of light absorption in human brain. Proc Natl Acad Sci USA 90: 3770-3774, 1993

4. Villringer A, Planck J, Hock C, Schleinkofer L, and Dirnagl U: Near infrared spectroscopy (NIRS): a new tool to study hemodynamic changes during activation of brain function in human adults. Neurosci Lett 154: 101-104, 1993

5. Hoshi $Y$ and Tamura M: Detection of dynamic changes in cerebral oxygenation coupled to neuronal function during mental work in man. Neurosci Lett 150: 5-8, 1993

6. Kato T, Kamei A, Takashima S, and Ozaki T: Human visual cortical function during photic stimulation monitoring by means of near-infrared spectroscopy. J Cereb Blood Flow Metab 13: 516-520, 1993

7. Eda H, Oda I, Ito $Y$, Wada $Y$, Oikawa $Y$, Tsunazawa $Y$, Takada M, Tsuchiya Y, Yamashita Y, Oda M, Sassaroli A, Yamada $Y$, and Tamura M: Multichannel time-resolved optical tomographic imaging system. Rev Sci Instrum 70: 3595-3602, 1999

8. Schmidt FE, Fly ME, Hillman EM, Hebden JC, and Delpy DT: A 32-channel time-resolved instrument for medical optical tomography. Rev Scientific Instrum 71: 256-265, 2000
9. Benaron DA, Vanhouten JP, Cheong W, Kermit EL, and King RA: Early clinical results of time-of-flight optical tomography in a neonatal intensive care unit. Proc SPIE 2389: 582-596, 1995

10. Barbour RL, Graber HL, Pei Y, Zhong S, and Schmitz $\mathrm{CH}$ : Optical tomographic imaging of dynamic features of dense-scattering media. J Opt Soc Am A Opt Image Sci Vis 18: 3018-3036, 2001

11. Maki A, Yamashita $Y$, Ito $Y$, Watanabe E, Mayanagi $Y$, and Koizumi $\mathrm{H}$ : Spatial and temporal analysis of human motor activity using noninvasive NIR topography. Med Phys 22: 1997-2005, 1995

12. Chance B, Anday E, Nioka S, Zhou S, Hong L, Worden K, Li C, Murray T, Ovetsky Y, Pidikiti D, and Thomas R: A novel method for fast imaging of brain function, noninvasively, with light. Opt Express 2: 411-423, 1998

13. Eda H, Sase I, Seiyama A, Tanabe HC, Imaruoka T, Tsunazawa Y, and Yanagida T: Optical topography system for functional brain imaging: mapping human occipital cortex during visual stimulation. Proceeding of Inter-Institute Workshop on In Vivo Optical Imaging, $\mathrm{p}$ 93-99, 2000

14. Wobst $P$, Wenzel $R$, Kohl $M$, Obrig $H$, and Villringer $A$ : Linear aspects of changes in deoxygenated hemoglobin concentration and cytochrome oxidase oxidation during brain activation. Neuroimage 13: 520-530, 2001

15. Wolf M, Wolf $U$, Toronov $V$, Michalos A, Paunescu LA, Choi $\mathrm{JH}$, and Gratton E: Different time evolution of oxyhemoglobin and deoxyhemoglobin concentration changes in the visual and motor cortices during functional stimulation: a near-infrared spectroscopy study. Neuroimage 16: 704-712, 2002

16. Sase I, Eda H, Seiyama A, Tanabe HC, Takatsuki A, and Yanagida T: Multi-channel optical mapping: Investigation of depth information. Proc SPIE 4250: 29-36, 2001

17. Toronov V, Webb A, Choi JH, Wolf M, Michalos A, Gratton $E$, and Hueber D: Investigation of human brain hemodynamics by simultaneous near-infrared spectroscopy and functional magnetic resonance imaging. Med Phys 28: 521-527, 2001

18. Strangman G, Culver JP, Thompson JH, and Boas DA: A quantitative comparison of simultaneous BOLD fMRI and NIRS recordings during functional brain activation. Neuroimage 17: 719-731, 2002

19. Miyai I, Tanabe HC, Sase I, Eda H, Oda I, Konishi I, Tsunazawa Y, Suzuki T, Yanagida T, and Kubota K: Cortical mapping of gait in humans: a near-infrared spectroscopic topography study. Neuroimage 14: 11861192, 2001

20. Baird AA, Kagan J, Gaudette T, Walz KA, Hershlag N, and Boas DA: Frontal lobe activation during object permanence: data from near-infrared spectroscopy. Neuroimage 16: 1120-1125, 2002

21. Pena M, Maki A, Kovacic D, Dehaene-Lambertz G, Koizumi $\mathrm{H}$, Bouquet $F$, and Mehler J: Sounds and silence: an optical topography study of language recognition at birth. Proc Natl Acad Sci USA 100: 11702-11705, 2003

22. Taga G, Asakawa K, Maki A, Konishi Y, and Koizumi H: Brain imaging in awake infants by near-infrared optical topography. Proc Natl Acad Sci USA 100: 


\section{A. TAKATSUKI et al.}

10722-11727, 2003

23. Noguchi $Y$, Watanabe E, and Sakai KL: An event-related optical topography study of cortical activation induced by single-pulse transcranial magnetic stimulation. Neuroimage 19: 156-162, 2003

24. Sevik EM and Chance B: Photon migration in a model of the head measured using time- and frequency-domain techniques: potentials of spectroscopy and imaging. Proc SPIE 1431: 84-96, 1991

25. Okada E, Firbank M, Schweiger M, Arridge SR, Cope $\mathrm{M}$, and Delpy DT: Theoretical and experimental investigation of near-infrared light propagation in a model of the adult head. Appl Opt 36: 21-31, 1997

26. Yamamoto T, Maki A, Kadoya T, Tanikawa Y, Yamad Y, Okada $\mathrm{E}$, and Koizumi $\mathrm{H}$ : Arranging optical fibres for the spatial resolution improvement of topographical images. Phys Med Biol 47: 3429-3440, 2002

27. Farrell TJ, Patterson MS, and Wilson B: A diffusion the- ory model of spatially resolved, steady-state diffuse reflectance for the noninvasive determination of tissue optical properties in vivo. Med Phys 19: 879-888, 1992

28. Arridge SR and Schweiger M: Photon-measurement density-functions. 2. Finite-element-method calculations. Appl Opt 34: 8026-8037, 1995

29. Okada E and Delpy DT: Near-infrared light propagation in an adult head model. I. Modeling of low-level scattering in the cerebrospinal fluid layer. Appl Opt 42: 2906-2914, 2003

30. Furutsu $\mathrm{K}$ and Yamada Y: Diffusion approximation for a dissipative random medium and the applications. Phys Rev E 50: 3634-3640, 1994

31. Gratton G, Maier JS, Fabiani M, Mantulin WW, and Gratton E: Feasibility of intracranial near-infrared optical scanning. Psychophysiology 31: 211-215, 1994

32. Worsley $\mathrm{KJ}$ and Friston $\mathrm{KJ}$ : Analysis of $\mathrm{fMRI}$ time-series revisited-again. Neuroimage 2: 173-181, 1995 